\title{
Associação entre Empatia e Personalidade em Estudantes de Medicina
}

\section{Association between Empathy and Personality in Medical Students}

\author{
Felipe Cesar de Almeida Claudino ${ }^{I}(\mathbb{D}$ \\ Marcelle Medeiros Lucena ${ }^{I}(\mathbb{C}$ \\ Enri Bobsin da Silva ${ }^{I} \mathbb{D}$ \\ Anne Baroffio ${ }^{I I}$ (iD) \\ Margaret Weidenbach Gerbase ${ }^{I, I} \mathbb{D}$
}

\section{PALAVRAS-CHAVE}

- Empatia.

- Personalidade.

- Educação Médica
${ }^{\mathrm{I}}$ Universidade Federal de Ciências da Saúde de Porto Alegre, Porto Alegre, Rio Grande do Sul, Brasil.

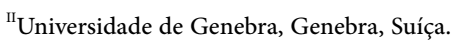




\section{KEYWORDS}

- Empathy.

- Personality.

- Medical Education.

Recebido em 20/11/19

Aceito em 31/8/20

\section{INTRODUÇÃO}

Sabe-se que o desempenho do médico é fortemente influenciado por seus conhecimentos, por suas competências clínicas e também pela capacidade de empatia. A empatia contribui positivamente para a relação médico-paciente e tem impacto na adesão ao tratamento ${ }^{1,2}$. Há ainda a evidência de que a habilidade empática pode ser influenciada pela personalidade ${ }^{3}$. Assim, das diversas características individuais do profissional que podem interferir no atendimento aos pacientes, destacamos neste artigo a personalidade e a empatia.

A personalidade é definida como um conjunto de características singulares de cada indivíduo. Embora possa ser modulada por diferentes contextos, a personalidade é referida como relativamente constante ao longo do tempo ${ }^{4}$, determinando um perfil único para cada pessoa constituído de diferentes características, aqui denominadas "domínios" ou "traços" de personalidade. Tais atributos podem ser influenciados pela cultura, educação e observação de comportamentos de outras pessoas ${ }^{5}$ que formam os domínios da personalidade e permeiam a conduta humana ${ }^{6}$.

A empatia, por sua vez, é um dos atributos mais destacados em profissionais da área da saúde, principalmente na relação médicopaciente, reunindo em seu conceito domínios cognitivos, emocionais e afetivos $^{7}$. Oriunda do grego, empatia significa a capacidade de colocar-se no lugar do outro, sem se confundir com ele ${ }^{8}$. Sendo assim, o uso dessa habilidade privilegia a escuta associativa, a atenção à linguagem corporal $\mathrm{e}$ a observação dos sinais de ansiedade ${ }^{9}$ do paciente pelo médico ${ }^{10}$. Estudos prévios demonstraram que a empatia tem impacto terapêutico positivo ${ }^{11,12}$ com aumento na satisfação do paciente, reduzindo a ansiedade e o sofrimento dele, com resultados clínicos significativamente melhores ${ }^{13}$. Sabe-se também que ela varia conforme os anos de experiência do profissional e com o gênero, e existe consenso na literatura de que as

\section{ABSTRACT}

Introduction: Empathy is a valuable attribute for a physician, as it positively influences the doctor-patient relationship. Previous studies indicate that there is a correlation between personality and empathy in medical students, but there is no data available in the Brazilian literature so far. This study aimed to analyze the empathy and personality traits of Brazilian medical students, and investigate their association. Methods: First year medical students from the 2015 and 2017 cohorts had their empathy and personality traits evaluated by two instruments using the following scales, respectively: Jefferson Scale of Empathy - Students version (JSE-S) and NEO-Five Factor Inventory (NEO-FFI). A descriptive analysis comprised mean, minimum and maximum scores, correlation coefficients and linear regression of personality and empathy. Results: 164 (96.4\%) students completed the surveys, and 50.5\% were women. The mean global score of the JSE-S was 117.6 \pm 10.9 ; stratification by gender showed scores of $119.5 \pm 10.5$ and $115.7 \pm 11.0$, respectively in women and men $(p<0.01)$. In the (he domain of 'conscientiousness' had the highest global mean $(29.1 \pm 3.8)$ and 'neuroticism', the lowest in 'open to new experiences' (20.6 \pm 3.3$)$. In men, the highest and the lowest scores were respectively in the domains 'conscientiousness' (29.4 \pm 3.9$)$ and 'neuroticism' (21.6 \pm 4.2$)$. There was a significant difference between men and women in the global score of the JSE-S, and in the personality domains of 'open to new experiences' and 'socialization'. Correlations between empathy and personality were found to be weak and not statistically relevant. Conclusion: The personality traits differed between men and women, but there was no significant correlation between empathy and personality among medical students enrolled in this cross-sectional study. Further investigations are needed to examine how empathy and personality modulate during medical studies using longitudinal approaches. 
Empathy - Students version (JSE-S) ${ }^{20}$ e o NEO-Five Factor Inventory (NEOFFI ${ }^{21}$. Esse último é composto pelos domínios de personalidade descritos por Costa e $\mathrm{McCrae}^{22}$, na denominada Teoria dos Cinco Fatores ou Big Five, definidos a partir de atributos, a saber: extroversão, socialização, conscienciosidade, neuroticismo e abertura para experiência ${ }^{23}$

A JSE-S é um questionário que avalia a empatia, amplamente validado e utilizado em estudantes de Medicina de diversas nacionalidades, sendo composto por 20 itens, dez com afirmativas diretas e dez com afirmativas reversas, cujas respostas variam em escala do tipo Likert de 1 ("discordo fortemente") a 7 ("concordo fortemente"). Os escores foram calculados respeitando a reversibilidade de cada item. Não há um ponto de corte, e, quanto maior for o escore obtido pelo estudante, maior será propensão à empatia.

O NEO-FFI, por sua vez, é um instrumento para avaliação de domínios individuais da personalidade, que são: extroversão, neuroticismo, conscienciosidade, socialização e abertura para experiência. O questionário é composto por 60 itens com escalas de 1 ("discordo fortemente") a 5 ("concordo fortemente"), com um total de 12 itens que avaliam cada um dos cinco domínios de personalidade. Os domínios podem ser assim descritos: a "extroversão" se refere à intensidade e frequência de relações interpessoais; a "socialização" se concentra nos tipos de interações sociais construídas pelo indivíduo; a "conscienciosidade" indica o grau de resiliência e motivação; o "neuroticismo" indica a adequação emocional; enquanto a "abertura à experiência" demonstra a disposição a novas experiências $^{24}$.

Um total de 77 alunos do ano de 2015 participaram da pesquisa, com adesão de $100 \%$ dos presentes no momento da enquete. No ano de 2017 , 93 alunos do primeiro ano foram convidados a participar, dos quais 87 responderam aos questionários $(93,5 \%)$. Nos dois grupos, observou-se um mínimo predomínio do sexo feminino, com 50,5\% em 2015 e 50,6\% em 2017.

Com o objetivo de unir os dados das duas coletas transversais, realizou-se uma análise preliminar em que se compararam a distribuição dos dados e os resultados das duas turmas de alunos com relação às variáveis analisadas. Observou-se uma distribuição não paramétrica nas duas turmas de alunos. A média da empatia do grupo 1 (77 alunos) foi de 118,77 \pm 9,9 e a média do grupo 2 (87 alunos) foi de 116,66 $\pm 11,73$. A comparação entre as médias dos escores obtidos para os parâmetros analisados entre os grupos foi realizada por meio do teste Mann-Whitney, não se observando diferença estatisticamente significativa $(\mathrm{p}=0,219)$.

A análise descritiva dos dados incluiu cálculos de média, escores mínimos e máximos, e desvio padrão de cada um dos domínios do NEO-FFI e da JSE-S. Na análise das variáveis contínuas, utilizaram-se os testes $\mathrm{t}$ de Student e Mann-Whitney e a regressão linear múltipla. A correlação entre personalidade e capacidade de empatia foi analisada por meio da correlação bivariada do índice de Spearman, após verificação de distribuição não paramétrica. $\mathrm{Na}$ análise de todos os dados, utilizou-se o software SPSS versão 22. A significância estatística foi estabelecida por meio de um valor de $\mathrm{p} \leq 0,05$.

O protocolo do estudo foi aprovado pelo Comitê de Ética em Pesquisa da Universidade Federal de Ciências da Saúde de Porto Alegre.

\section{RESULTADOS}

O total de alunos participantes foi de 164 (96,4\%). No questionário JSE-S, utilizado para avaliação da empatia, a média do escore global obtida foi de 117,6 $\pm 10,9$, observado na Tabela 1 . Na avaliação dos escores da JSE-S para empatia estratificada por sexo, observaram-se as seguintes médias: $119,5 \pm 10,5$ para o grupo feminino e $115,7 \pm 11$ para o masculino, com diferença significativa entre os grupos $(\mathrm{p}<0,01)$.

A análise dos domínios da personalidade demonstrou que a maior média global foi a de "conscienciosidade" (29,1 $\pm 3,8)$, e "neuroticismo" obteve a menor $(21,7 \pm 4,7)$, como se observa na Tabela 2. A maior pontuação obtida da personalidade no grupo feminino foi em "conscienciosidade", com média de $29,4 \pm 3,79$, e a menor em "abertura para experiência", com média de 20,6 $\pm 3,3$. No grupo masculino, "conscienciosidade" e "neuroticismo" obtiveram as maiores e menores médias (29,4 $\pm 3,9$ e 21,6 $\pm 4,2$, respectivamente). Constatou-se diferença significativa entre os grupos após estratificação no escore dos domínios de personalidade "abertura a novas experiências" e "socialização", conforme ilustra a Tabela 1.

Fez-se a avaliação da correlação pela média global e pela estratificação por sexo. Encontraram-se correlações fracas, e nenhuma delas foi estatisticamente significativa (Tabela 2).

A regressão linear múltipla não apresentou resultados significativos entre os domínios de personalidade e empatia, conforme mostra a Tabela 3.

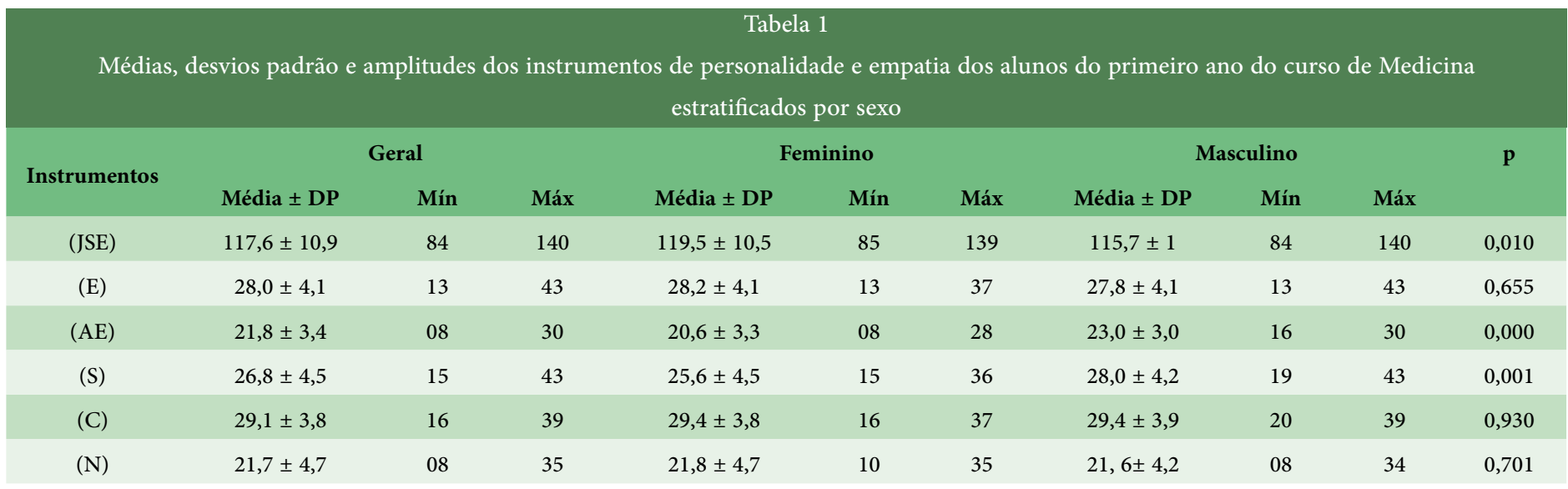

(JSE): Jefferson Scale Empathy; (E): extroversão; (AE): abertura para experiência; (S) socialização; (C) conscienciosidade; (N): neuroticismo; Mín: mínimo; Máx: máximo; Média \pm DP: média e desvio padrão.

Fonte: Elaborada pelos autores.

$3 \mid$\begin{tabular}{l|l} 
REVISTA BRASILEIRA DE EDUCAÇÃO MÉDICA \\
\hline 44 (4) : e150; 2020
\end{tabular} 


\begin{tabular}{|c|c|c|c|c|c|}
\hline \multicolumn{6}{|c|}{ Tabela 2} \\
\hline \multicolumn{6}{|c|}{ Correlação entre empatia (JSE-S) e personalidade (NEO-FFI) } \\
\hline JSE & $\mathbf{E}$ & AE & S & C & $\mathbf{N}$ \\
\hline Masculino & 0,203 & 0,004 & 0,073 & 0,050 & 0,028 \\
\hline Feminino & $-0,064$ & $-0,055$ & 0,046 & 0,108 & $-0,077$ \\
\hline Geral & 0,078 & $-0,071$ & $-0,006$ & 0,063 & $-0,007$ \\
\hline
\end{tabular}

E: extroversão; AE: abertura para experiência; S: socialização; C: conscienciosidade; N: neuroticismo.

Fonte: Elaborada pelos autores.

\section{Tabela 3}

Regressão linear múltipla hierarquizada de empatia e personalidade

\begin{tabular}{|cccc|}
\hline Variável independente & $(\mathbf{B})$ & Desvio padrão & $(\mathbf{p})$ \\
\hline Extroversão & 0,115 & 1,377 & 0,170 \\
\hline Abertura para experiência & $-0,117$ & $-1,368$ & 0,173 \\
\hline Conscienciosidade & 0,071 & 0,873 & 0,384 \\
\hline Neuroticismo & $-0,058$ & $-0,715$ & 0,476 \\
\hline Socialização & 0,074 & 0,893 & 0,373 \\
\hline & $\mathrm{R}$ & $\mathrm{R}^{2}$ & \\
\hline & 0,195 & 0,038 & \\
\hline
\end{tabular}

Fonte: Elaborada pelos autores.

\section{DISCUSSÃO}

O presente estudo teve como objetivo abordar a relação entre a empatia e a personalidade entre estudantes de Medicina do primeiro ano da graduação. Os nossos resultados demonstraram que os alunos com maior tendência à extroversão têm uma propensão significativamente maior à empatia, reforçando a hipótese de que essas duas características se relacionam ${ }^{3}$. Segundo Costa et at. ${ }^{16}$, que também avaliaram empatia e personalidade em estudantes de Medicina, a análise dos fatores de personalidade contribuiu para a identificação de alunos mais empáticos, sobretudo nas dimensões conscienciosidade e abertura a novas experiências.

Na nossa análise dos níveis de empatia, os maiores escores foram das mulheres, o que é reforçado pelos resultados da literatura sobre estudos realizados com grupos de estudantes de Medicina ${ }^{25}$. De acordo com o estudo de Duarte et al. ${ }^{26}$, as mulheres, no que concerne aos discentes de Medicina, são mais empáticas do que os homens, e essa diferença tente a crescer ao longo do curso ${ }^{26}$. Há outros aspectos que contribuem para a mudança dos níveis de empatia ao longo do curso de Medicina além da personalidade e do gênero. Piumatti et al. ${ }^{27}$ destacam, por exemplo, que a motivação para o estudo da Medicina centrada no paciente pode contribuir para manter a empatia ao longo da formação médica e, por isso, deve ser estimulada.

Uma revisão da literatura realizada por Fragkos ${ }^{28}$ mostrou que as intervenções de empatia em estudantes de Medicina são eficazes e que diferentes medidas têm sido adotadas para o estímulo precoce dessa habilidade. Exemplo disso se observa no estudo realizado por Schweller, Costa et al. $^{29}$, no qual a aprendizagem por meio de consultas com pacientes simulados favoreceu a empatia de estudantes em diferentes anos do curso de Medicina ${ }^{29}$. Salientamos também a importância de estratégias de aprendizagem precoces para o desenvolvimento da empatia nos estudantes de Medicina, pois, como ressaltam Piumatti et al. ${ }^{27}$, alunos com menores níveis de empatia tendem a reduzir ainda mais seu escore com o passar do tempo. Assim, é possível individualizar intervenções pedagógicas integradas ao currículo para mudar esse cenário.

$\mathrm{Na}$ análise da avaliação da personalidade, por sua vez, observamos que a conscienciosidade é o traço predominante entre os cinco domínios avaliados nos dois sexos. O mesmo achado foi descrito por Lievens et al. $^{30}$, entre outros autores, que destacam a conscienciosidade como um fator positivo no desempenho durante o curso de Medicina, sugerindo que os alunos com essa qualidade são mais propensos à proatividade, à autodisciplina e à menor tendência à desistência do curso. Outro estudo, realizado por $\mathrm{Laher}^{31}$, que avaliou a personalidade de acordo com o gênero, observou que os domínios relativos à abertura à experiência $\mathrm{e}$ à capacidade de socialização foram maiores nos homens, assim como descrito em nossos resultados. Esses atributos, como a abertura para experiência, demonstram uma maior flexibilidade, criatividade e também a capacidade de tomada de perspectiva diferente dos demais colegas, enquanto a socialização reflete uma maior capacidade de interação, tendo uma correlação positiva e significativa com a empatia ${ }^{3}$. A extroversão, como apontado anteriormente, está relacionada a uma maior capacidade de empatia, e, nos nossos achados, o escore desse domínio foi maior nas mulheres. Por fim, o neuroticismo também foi maior nas mulheres, e há a evidência de que o aumento dessa característica da personalidade ao longo do curso está associado a um melhor desempenho acadêmico ${ }^{32}$.

O conhecimento acerca da personalidade do estudante de Medicina vai além da capacidade de predizer a empatia e sua trajetória ao longo da graduação. Segundo Mullola et al..$^{33}$, os traços de personalidade podem influenciar na carreira médica, desde a escolha da especialidade até a relação profissional com o paciente. Portanto, ao mesmo tempo que a formação para a prática médica com estratégias de ensino-aprendizagem adequadas pode favorecer o desenvolvimento da empatia, também poderá reforçar os traços de personalidade, permitindo aos alunos a escolha adequada da sua prática futura entre as diversas especialidades médicas.

Diante do exposto, destacamos dois pontos fortes do estudo: o primeiro foi a uniformidade da distribuição de sexo nas duas enquetes, sendo relevante para a análise dos resultados obtidos, já que a literatura demonstra haver uma diferença em aspectos da empatia no que se refere à comparação entre os $\operatorname{sexos}^{25}$. O segundo ponto foi a alta taxa de adesão, pois os discentes demonstraram, por meio da sua participação, o interesse no estudo do perfil do estudante de Medicina da instituição. Como limitação, temos um estudo transversal realizado por dois grupos em momentos distintos, em uma única instituição, e a utilização de escalas autorreferidas, que podem não refletir a empatia na relação médico-paciente. A amostra de 164 estudantes pode não ser representativa da população geral de estudantes de Medicina, necessitando de grupos multicêntricos.

Por fim, faz-se fundamental investigar como a relação entre personalidade e empatia se comporta no tempo, sobretudo se a capacidade de empatia se modifica ao longo dos anos de formação médica e qual a relação desta com a modulação nos traços de personalidade ${ }^{34}$, assim como outras características que possam interferir na empatia, como ambiente de aprendizagem e qualidade de vida ${ }^{35}$. Assim, a análise da evolução longitudinal desses grupos poderá avaliar potenciais preditores de empatia ao longo do curso de Medicina.

REVISTA BRASILEIRA DE EDUCAÇão MÉDICA

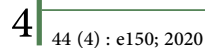




\section{CONCLUSÃO}

Os resultados deste estudo demonstram que, entre os cinco domínios de personalidade analisados, a conscienciosidade predomina tanto nos alunos do sexo masculino como no feminino; esse traço de personalidade se relaciona à autodisciplina e à proatividade. Além do mais, observouse diferença significativa nos domínios denominados socialização e abertura para experiência, os quais foram mais expressivos nos homens, comparativamente às mulheres. Já os domínios de neuroticismo e extroversão predominaram nas mulheres com relação aos homens, mas a diferença não alcançou significância estatística. Os escores de empatia, por sua vez, foram significativamente maiores em mulheres, condizente com os achados da literatura. Há evidências da relação entre personalidade e empatia, e medidas de intervenção que reforcem traços positivamente associados a empatia podem ser realizadas ao longo da formação médica. Estudos complementares com desenho longitudinal permitirão elucidar as inter-relações entre personalidade e empatia, assim como acompanhar a potencial modulação entre elas na prática clínica e também analisar outros fatores que afetem a empatia, além da personalidade.

\section{REFERÊNCIAS}

1. Bensing JM, Dronkers J. Instrumental and affective aspects of physician behavior. Med Care. 1992;30(4):283-98.

2. Hojat M, Louis DZ, Maio V, Gonnella JS. Empathy and health care quality. Am J Med Qual. 2013;28(1):6-7.

3. Abe K, Niwa M, Fujisaki K, Suzuki Y. Associations between emotional intelligence, empathy and personality in Japanese medical students. BMC Med Educ. 2018;18(1): 1-9 [acesso em 29 set 2019]. Disponível em: https:// bmcmededuc.biomedcentral.com/articles/10.1186/s12909-018-1165-7.

4. Allport GW. Personalidade padrões e desenvolvimento. São Paulo: Editora da Universidade de São Paulo; 1996.

5. Linton R. O homem: uma introdução à antropologia. São Paulo: Livraria Martins; 1962.

6. McCrae RR, John OP. An introduction to the five-factor model and its applications. J Pers. 1992;60(2):175-215.

7. Mehrabian A, Young AL, Sato S. Emotional empathy and associated individual differences. Current Psychol. 1988;7(3):221-40.

8. Soar FEJ. A interação médico-cliente. Rev Assoc Med Bras. 1998.44(1):3542 [acesso em 30 ago 2019]. Disponível em: http://www.scielo.br/scielo. php?script=sci_arttext\&pid=S0104-42301998000100007\&lng=en.

9. Halpern J. What is clinical empathy? J Gen Intern Med. 2003;18(8):670-4.

10. Suchman AL, Markakis K, Beckman HB, Frankel R. A model of empathic communication in the medical interview. JAMA. 1997;277(8):678-82.

11. Halpern J. Empathy and patient-physician conflicts. J Gen Intern Med. 2007;22(5):696-700.

12. Flickinger TE, Saha S, Roter D, Korthuis PT, Sharp V, Cohn J, et al. Clinician empathy is associated with differences in patient-clinician communication behaviors and higher medication self-efficacy in HIV care. Patient Educ Couns. 2016;99(2):220-6.

13. Derksen F, Bensing J, Lagro-Janssen A. Effectiveness of empathy in general practice: a systematic review. Br J Gen Pract. 2013;63(606):76-84.

14. Nascimento HCF, Ferreira WAF, Silva AMTC, Carvalho IGM, Bastos GCFC, Almeida RJ. Análise dos níveis de empatia de estudantes de Medicina. Rev Bras Educ Med. 2018;42(1):152-60.

15. Song Y, Shi M. Associations between empathy and big five personality traits among Chinese undergraduate medical students. PLoS One. 2017;12(2):1-13 [acesso em 16 maio 2019]. Disponível em: https:// journals.plos.org/plosone/article?id=10.1371/journal.pone.0171665.

16. Costa P, Alves R, Neto I, Marvão P, Portela M, Costa MJ. Associations between medical student empathy and personality: a multiinstitutional study. PLoS One. 2014;9(3):1-7 [acesso em 22 maio 2019]. Disponível em: https://journals.plos.org/plosone/article?id=10.1371/ journal.pone.0089254.

17. Magalhães E, Costa P, Costa MJ. Empathy of medical students and personality: evidence from the Five-Factor Model. Med Teach. 2012;34(10):807-12.

18. Hojat M, Vergare M, Isenberg G, Cohen M, Spandorfer J. Underlying construct of empathy, optimism, and burnout in medical students. Int J Med Educ. 2015;6:12-6.

19. Ertram K, Randazzo J, Alabi N, Levenson J, Doucette JT, Barbosa P. Strong correlations between empathy, emotional intelligence, and personality traits among podiatric medical students: a cross-sectional study. Educ Health. 2016;29(3):186-94.

20. Paro HB, Daud-Gallotti RM, Tibério IC, Pinto RM, Martins MA. Brazilian version of the Jefferson Scale of Empathy: psychometric properties and factor analysis. BMC Med Educ. 2012; 73(12):1-7.

21. Magalhães E, Salgueira A, Gonzalez AJ, Costa JJ, Costa MJ, Costa P, et al. NEO-FFI: psychometric properties of a short personality inventory in Portuguese context. Psicol Reflex Crit. 2014;27(4):642-57.

22. Costa PT, McCrae RR. Revised NEO personality inventory (NEOPI-R) and NEO five-factor inventory (NEO-FFI) professional manual. Odessa: Psychological Assessment Resources;1992.

23. Gomes CMA, Golino HS. Relações hierárquicas entre os traços amplos do Big Five. Psicol Reflex Crit. 2012;25(3):445-56.

24. Nunes CHSS, Hutz CS. O modelo dos Cinco Grandes Fatores de Personalidade. In: R. Primi, organizador. Temas em avaliação psicológica. São Paulo: Casa do Psicólogo; 2002.

25. Kataoka HU, Koide N, Ochi K, Hojat M, Gonnella JS. Measurement of empathy among Japanese medical students: psychometrics and score differences by gender and level of medical education. Acad Med. 2009;84(9):1192-7.

26. Duarte MIF, Raposo MLB, Rodrigue PJFSF, Branco MC. Measuring empathy in medical students, gender differences and level of medical education: an identification of a taxonomy of students. Investigación Educ Médica. 2016;5(20):253-60.

27. Piumatti G, Abbiati M, Baroffio A, Gerbase MW. Empathy trajectories throughout medical school: relationships with personality and motives for studying medicine. Adv in Health Scie Educ. 2020. [acesso em 29 abr 2020]. Disponível em https://link.springer.com/article/10.1007/ s10459-020-09965-y.

28. Fragkos KC, Crampton PES. The Effectiveness of Teaching Clinical Empathy to Medical Students: A Systematic Review and MetaAnalysis of Randomized Controlled Trials. Acad Med. 2019. [acesso 19 abril 2020]. Doi: 10.1097/ACM.0000000000003058.

29. Schweller M, Costa FO, Antônio MA, Amaral EM, Carvalho-Filho MA. The impact of simulated medical consultations on the empathy levels of students at one medical school. Acad Med. 2014;89(4):632-7.

30. Lievens F, Coetsier P, Fruyt FD, Maeseneer J. Medical students' personality characteristics and academic performance: a five-factor

$5 \mid$\begin{tabular}{l|l} 
REVISTA BRASILEIRA DE EDUCAÇÃO MÉDICA \\
\hline 44 (4) : e150; 2020
\end{tabular} 
model perspective. Med Educ. 2002;36(11):1050-6.

31. Laher S. Structural equivalence and the NEO-PI-R: implications for the applicability of the five-factor model of personality in an African context: theoretical research. J Ind Psychol. 2008;34:76-80.

32. Lourinho I, Ferreira MA, Severo M. Personality and achievement along medical training: Evidence from a cross-lagged analysis. PLoS One [online]. 2017;12(10):e0185860 [acesso em 12 abr 2020]. Disponível em: https://doi.org/10.1371/journal.pone.0185860.

33. Mullola S, Hakulinen C, Presseau J, Porras DGR, Jokela M, Hintsa $\mathrm{T}$, et al. Personality traits and career choices among physicians in Finland: employment sector, clinical patient contact, specialty and change of specialty. BMC Med Educ. 2018;18(1):52 [acesso em 21 abr 2020]. Disponível em: https://bmcmededuc.biomedcentral.com/ articles/10.1186/s12909-018-1155-9.

34. Costa FD, Azevedo RCS. Empatia, relação médico-paciente e formação em medicina: um olhar qualitativo. Rev Bras Educ Med. 2010;34(2):261-9.

35. Paro HBMS, Silveira PSP, Perotta B, Gannam S, Enns SC, Giaxa RRB, et al. Empathy among Medical Students: Is There a Relation with Quality of Life and Burnout? PLoS One. 2014;9(4):1-10 [acesso em 18 abr 2019]. Disponível em: https://journals.plos.org/plosone/ article?id=10.1371/journal.pone.0094133.

\section{CONTRIBUIÇÃO DOS AUTORES}

Felipe Cesar de Almeida Claudino contribuiu com a aplicação dos questionários, tabulação dos resultados, análise dos resultados e a produção do artigo. Marcelle Medeiros Lucena contribuiu com a aplicação dos questionários, tabulação dos resultados e produção do artigo. Enri Bobsin da Silva contribuiu com a aplicação dos questionários, tabulação dos resultados e produção do artigo. Anne Baroffio contribuiu com a concepção do projeto, coorientação e produção do artigo. Margaret Weidenbach Gerbase contribuiu com a concepção do projeto, seleção dos questionários, aplicação dos protocolos, orientação principal, análise dos resultados e produção do artigo.

\section{CONFLITO DE INTERESSES}

Os autores declaram não haver conflito de interesses nesse estudo.

\section{ENDEREÇO PARA CORRESPONDÊNCIA}

Margaret Weidenbach Gerbase. Universidade Federal de Ciências da Saúde de Porto Alegre, Curso de Medicina, Departamento de Clínica Médica, Rua Sarmento Leite, 245, $6^{\circ}$ andar, sala 611, Porto Alegre, RS, Brasil. CEP: 90050-170.

E-mail: gerbase@ufcspa.edu.br 